\title{
A systematic review to identify whether perfusate biomarkers produced during hypothermic machine perfusion can predict graft outcomes in kidney transplantation
}

\author{
Francesco Guzzi, MD ${ }^{1,2}$, Simon R. Knight, FRCS, PhD ${ }^{1}$, Rutger J. Ploeg, MD, PhD ${ }^{1,3}$, \\ James P. Hunter, FRCS, $\mathrm{PhD}^{1}$
}

\footnotetext{
${ }^{1}$ Nuffield Department of Surgical Sciences, University of Oxford and Oxford Transplant Centre, Churchill Hospital, Oxford, United Kingdom

${ }^{2}$ Department of Experimental and Clinical Biomedical Sciences “Mario Serio”, University of Florence, Florence, Italy

${ }^{3}$ Oxford Biomedical Research Centre, Oxford, United Kingdom
}

Correspondence information

Francesco Guzzi, MD

Dept. of Experimental and Clinical Biomedical Sciences

University of Florence

Viale Pieraccini 6, 50139, Firenze, Italy

E-mail: francesco.guzzi@gmail.com 


\section{Authorship}

\section{Authorship statement}

All authors made a significant contribution to the content of this manuscript as per ICMJE recommendations. F.G., R.J.P. and J.P.H. conceived the research idea. F.G. participated in the literature systematic research, participated in the eligibility screening and full text articles revision, participated in articles evaluation and data collection and wrote the article. S.R.K. provided support to the systematic research and to the evaluation process and critically reviewed the article. R.J.P. provided support to the systematic research and to the evaluation process and critically reviewed the article. J.P.H. participated in the literature systematic research, participated in the eligibility screening and full text articles revision, participated in articles evaluation and data collection and critically reviewed the article.

\section{Disclosures}

The authors declare no conflicts of interest.

\section{Funding}

No funding was received for the conduct of this research.

\section{Keywords}

Kidney transplantation; Hypothermic Machine Perfusion; Biomarkers; Allograft outcomes; Systematic review. 


\section{Abbreviations}

AKI, acute kidney injury

Ala-Ap, alanine-aminopeptidase

AST, aspartate transaminase

AUC, area under the curve

DBD, donation after brain death

DCD, donation after circulatory death

DGF, delayed graft function

eGFR, estimated glomerular filtration rate

EVNP, ex vivo normothermic perfusion

FABP, fatty acid binding protein (H-heart, L-liver)

GST, glutathione S-transferase (t-total, a-alpha, p-pi)

HMP, hypothermic machine perfusion

H-NMR, proton nuclear magnetic resonance

iCa, ionized calcium

IL-18, interleukin-18

KIM-1, kidney injury molecule-1

LDH, lactate dehydrogenase

LPOPs, lipid peroxidation products

MDA, malondialdehyde

miR-21, mirco-RNA 21

MMP, matrix metalloproteinase

NAG, n-acetylglucosamine

NGAL, neutrophil gelatinase-associated lipocalin 
NMP, normothermic machine perfusion

NPV, negative predictive value

OR, odds ratio

PNF, primary non-function

PPV, positive predictive value

RCT, randomized clinical trial

ROC, receiver operator curve

SCS, static cold storage

UW, University of Wisconsin 


\begin{abstract}
There is good evidence to support the use of Hypothermic Machine Perfusion (HMP) over static cold storage as the favoured preservation method for deceased donor kidneys. However, the utility of HMP as a tool to assess the viability of kidneys for transplant is unclear. There is a need to determine whether perfusate biomarkers produced during HMP can predict post-transplant outcomes and assess the suitability of organs for transplantation. Three different Databases (MEDLINE, Embase, Transplant Library) were screened to 31 May 2019. Articles were included if a relationship was reported between one or more perfusate biomarkers and post-transplant outcomes. Studies were assessed and graded for methodological quality and strength of evidence. Glutathione S-transferase was the most promising biomarker for predicting delayed graft function, but its predictive ability was at best moderate. Analysis of primary non-function rates was challenging due to low occurrence rates and small sample sizes. Existing studies are limited in quality and have not yielded biomarkers for kidneys undergoing HMP that are able to predict post-transplant outcomes with sufficient accuracy to support routine clinical use. Further studies with larger samples and more robust methodology are needed.
\end{abstract}

(PROSPERO registration: CRD42019121161) 


\section{Introduction}

Kidney transplantation is the treatment of choice for the majority of patients with end stage kidney disease, improving both quality of life and survival. However, the shortage of deceased donor organs leads to long waiting times and there is a need to identify strategies to increase the donor pool. Increasing evidence suggests that Hypothermic Machine Perfusion (HMP) is the best preservation method compared with simple Static Cold Storage (SCS) for extended criteria donor and older kidneys. ${ }^{1,2}$ HMP reduces the rate of delayed graft function (DGF) and increases 1-year graft survival in these groups when a higher risk donor organ was used and may also improve outcomes in all donor types. ${ }^{3}$ HMP has become standard practice in some centres and also in some countries i.e. in The Netherlands, where all deceased donor kidneys are placed on an HMP device at the retrieval centre. While perfusion will allow better assessment of viability during the period when bridging from donor to recipient, the role of perfusate biomarkers produced during HMP prior to implant remains unclear yet. There is a clear need for better and more objective markers of viability to reduce discard rates on the one hand but also avoid transplantation of poor-quality kidneys. ${ }^{4}$ Better assessment will help to obtain improved outcomes and survival after transplantation.

A systematic review published in 2012 assessed the role of biomarkers in HMP perfusate and in the donor urine. ${ }^{5}$ This review was limited by the small overall number and even smaller number of good quality studies included, although it did report a link between rates of delayed graft function (DGF) and perfusate levels of lactate dehydrogenase (LDH), glutathione Stransferase (GST) and aspartate transaminase (AST). The authors also reported a significant association between primary non-function (PNF) and GST (in 2 out of 3 studies) as well as LDH (1/3), with no association found between any biomarkers and graft survival. In the last seven years, there have been further publications on the impact of biomarkers related to organ viability 
during HMP. In this study we have undertaken a systematic review of the current literature to update findings and to consider the role of new biomarkers reflecting kidney injury. The objectives of this review are to assess whether perfusate biomarkers can predict post-transplant outcomes (namely DGF, PNF and graft survival) and to determine the strength of the biomarkers' association with outcome. In addition, we aim to establish whether there are any differences between biomarkers in the DBD and DCD settings.

\section{Materials and Methods}

Reporting of this review follows the Preferred Reporting Items for Systematic Reviews and Meta-analysis (PRISMA) guidelines. The analysis methods, search strategies and inclusion criteria were specified in advance and documented in a shared protocol. The review protocol was prospectively registered with PROSPERO and can be found online (registration number: CRD42019121161).

\section{Literature search}

MEDLINE (1946 - present), Embase (1974 - present) and the Transplant Library (2004 present) were searched through the Ovid platform for studies comparing HMP perfusate biomarker levels and outcomes after transplantation. Outcomes of interest included DGF (usually defined as the need for one or more haemodialysis sessions within the first week post transplantation, as opposed to immediate graft function), PNF (defined by permanent need for haemodialysis after transplantation or the failure of resolution of DGF) and long-term outcomes including graft function (serum creatinine or eGFR) and graft survival. All articles published before the $31^{\text {st }}$ May 2019 were considered. The search terms are detailed in the search strategy 
document (Table S1). Reference lists were scanned for relevant articles overlooked by the literature search.

\section{Inclusion/exclusion criteria}

Eligibility screening by title and abstract was independently performed by two authors (FG, $\mathrm{JH}$ ) and any discrepancies were discussed and resolved. Studies were included if they utilised hypothermic machine perfusion in human kidneys intended for transplantation, and related perfusate biomarkers to one or more of the following clinical outcomes: graft survival; PNF; DGF and graft function. Biomarkers were always measured from perfusate samples, drawn at various time point after perfusion start. Abstracts, reviews and commentaries were excluded. If there were no biomarker analyses or transplant outcomes, or no association between them was reported, the articles were excluded. Duplicates still present were identified by juxtaposing author names, articles titles, and affiliations. The remaining articles were independently reviewed by two investigators (FG, JH). Articles from the same research groups considering overlapping populations were included and grouped to easily detect the most recent results for each outcome of interest. Data regarding the study characteristics were collected by one author (FG) with the support of an extraction table previously refined by all authors. A second author (JH) subsequently checked all the extracted data and disagreements were resolved by discussion between all the authors.

\section{Study evaluation}

Data regarding the strength of relationship between biomarkers and perfusion parameters and clinical outcomes including receiver operator curve (ROC) area under the curve (AUC), sensitivity, specificity, positive predictive value, negative predictive value and correlation coefficients were extracted and each article was graded according to the strength of evidence. 
Strength was determined by the breadth of the confidence intervals and the method of statistical analysis. Biomarker thresholds were considered when present and articles were assessed for sensitivity and specificity and evidence of biomarker validation. Due to the limited amount of available data, a narrative synthesis was employed to discuss the results.

Each study was evaluated for risk of bias using a modified version of the checklist by Moga et al. ${ }^{6}$ This is a quality appraisal tool for case series developed in 2012 by the Institute of Health Economy of Alberta (Canada). Since no specific interventions were evaluated and no adverse events were expected, a maximum of 15 points were assigned to each paper consequently graded as “good” (14-15), “fair” (11-13,5) or “poor” $(<11)$.

\section{Results}

Included studies

Literature searches identified a total of 552 citations. After eliminating duplicates, 450 remained. Of these, 390 were discarded after evaluating title and abstract for eligibility. Full text versions of the remaining 60 articles were reviewed to ensure they met the inclusion criteria. 31 studies were excluded following review as detailed in the search flow diagram (Figure 1) and 29 articles published from 1973 to 2017 were identified for inclusion. Articles from the same research groups and/or considering overlapping populations were included, and consequently flagged, so important findings were not overlooked.

\section{Study characteristics}

The most relevant characteristics of each study are summarised in Table 1. The included studies were heterogeneous in terms of sample size with the largest presenting data from 670 and the smallest from 11 transplants. Of the 29 studies, only two analysed data collected from a 
randomised controlled trial from Moers et al. ${ }^{1}$ (MP-Trial). Both were secondary analyses from the machine perfused arm of the trial, whose primary objective was to establish the ability of HMP to reduce DGF occurrence. Both were included in our review: Moers et al. ${ }^{7}$ considered all the kidneys from the trial and Nagelschmidt et al. ${ }^{8}$ limited the analysis to only DBD donors older than 55 years. Two additional studies, Hall et al. ${ }^{9}$ and Parikh et al. ${ }^{10}$, collected data from a large prospective multi-centre cohort $(\mathrm{n}=671)$. Data from an additional two studies were prospectively collected, not randomised nor blinded and limited by a small sample size (11 to 50 transplants). The remaining were retrospective studies, of these, the work by Hoogland et al. ${ }^{11}(\mathrm{n}=335)$, de Vries et al. ${ }^{12}(\mathrm{n}=231)$ and van Smaalen et al. ${ }^{13}(\mathrm{n}=390)$ had large sample sizes and scored "good/fair” overall for quality.

The majority of the included studies were conducted in Europe (22/29) and almost a third were published in the last 5 years. Sixteen studies considered grafts from either DCD (10/16) or DBD (6/16) donors; the remaining studies evaluated grafts from a mixed, mainly DBD donors $(n=7)$, or mainly DCD donors $(n=3)$. In three studies the donor characteristics were not detailed.

\section{Quality assessment}

According to the quality checklist, the four previously mentioned prospective studies (Moers et al.; Nagelschmidt et al.; Hall et al.; Parikh et al. $)^{7-10}$ were graded as of "good" quality, 11 articles graded as "fair" and 14 as "poor". Detailed results from the quality scoring/risk of bias are reported (Table S2).

\section{Biomarkers}

The main results from each study are outlined in Table 2. Glutathione S-transferase (GST) as total-GST (t-GST) or its isoforms (alpha-GST and pi-GST), was the most commonly evaluated biomarker (14/29), followed by lactate dehydrogenase (LDH) (13/29) and lactate levels (9/29). 
Fatty acid binding protein (FABP, in its isoforms heart-FABP and liver-FABP, 4/29), neutrophil gelatinase-associated lipocalin (NGAL, 3/29), interleukin-18 (IL-18, 2/29) lipid peroxidation products (LPOPs, 2/29) and perfusate ionized calcium (iCa, 2/29) were considered in at least two articles from different authors. Single-study evaluations were present for: histones H3, kidney injury molecule-1 (KIM-1), aspartate transaminase (AST), alanine-aminopeptidase (Ala-Ap), matrix metalloproteinase 9 and 2 (MMP-9, MMP-2), n-acetylglucosamine (NAG), redox-active iron, mirco-RNA 21 (miR-21), and perfusate proton nuclear magnetic resonance (H-NMR) spectroscopy.

\section{Outcomes}

The most commonly evaluated outcome was delayed graft function (23/29). Only twelve articles provided data on primary non-function. Finally, nine articles studied the relationship between one or more biomarker levels and long-term graft function or patients' survival up to 5year post transplantation.

Glutathione S-transferase. GST was the most studied and the most promising biomarker, particularly for DGF prediction, confirming the previous finding by Bhangoo et al. ${ }^{5}$ To our knowledge, the first attempt to establish a correlation between GST levels during hypothermic machine perfusion and transplant outcome in the clinical setting was published in $1981 .{ }^{14}$ In this pioneering work, Cho et al. established a statistically significant correlation between GST (previously known as ligandin) levels at the end of perfusion and the development of acute tubular necrosis defined as the need of one haemodialysis session in the first three days posttransplantation. GST levels increased throughout perfusion in all the included studies and they were associated with DGF in 7/10 articles three of which were "good” quality studies with two (Moers et al. ${ }^{7}$ and Hall et $a l^{9}{ }^{9}$ ) demonstrating a significant association after multivariable 
analysis. However, the strength of the association of GST levels and DGF was less convincing and the different isoforms and assays used meant that direct comparison of values between studies was not possible. ROC analysis in the Moers paper based on GST levels at the end of perfusion was 0.67, in the Hall paper the authors demonstrate unadjusted ROC of 0.61 and ROC of 0.70 when adjusted for donor, transport and recipient variables. These values were based on the "log of the optimal cut off value". In the Nagelschmidt paper ${ }^{8}$ a cut off of $10 \mathrm{ug} / \mathrm{ml}$ of alphaGST had a positive predictive value (PPV) of $71.4 \%$ and a negative predictive value (NPV) of 77.9\% for DGF. For t-GST, a cutoff of 450 U/L yielded a PPV of only $37.5 \%$ and a NPV of 79.1\%. When data were available, a statistically significant association between elevated GST levels and PNF was reported in 6/8 papers, but not confirmed in the population studied by Moers et al. and Nagelschmidt et al. Moreover, four of these articles reporting a positive association were all performed by the University of Maastricht group with data collected between 1993 and 1997. Significant association of GST levels with long-term outcome has never been reported.

Lactate dehydrogenase. Our results show that LDH elevation was associated with DGF in 8/11 articles, but only in Hoogland et al. ${ }^{11}$ was the association maintained after multivariable analysis. However, the strength of the association was weak (OR, $1.002(1.001-1.004) \mathrm{p}=0.007)$. The association with PNF was again less common with a significant correlation found in only 2/8 papers, both from the Maastricht group (Hoogland et al. ${ }^{11}$ and de Vries et al. ${ }^{12}$ ). Again the strength of the association was weak with ROC 0.699 and the transferability of these data to future populations is unclear as the PNF rate in the Hoogland study was unusually high at $20 \%$. The only positive association between elevated LDH levels and long-term outcome was reported, again by Hoogland et al., with 1-year eGFR, but not with graft survival. 
Lactate. The association between elevated lactate levels and DGF/PNF was repeatedly investigated, mostly in the older articles. In total, 4/8 papers found a significant association between lactate levels and the occurrence of DGF, although there was some overlap between similar populations as highlighted in Table 1. This association was not maintained after multivariable analysis in any of the included studies. Lactate levels were never found to be associated with PNF (0/2) or long-term outcomes (0/1).

FABP, LPOPs and IL-18. FABP (with its isoforms H-FABP and L-FABP), LPOPs and IL18 were reported to be significantly associated with DGF respectively in 2/3, 2/2 and 2/2 articles. H-FABP was identified, together with GST and NAG, by Moers et al. ${ }^{7}$ as an independent predictor for DGF with a moderate prognostic value (AUC of 0.64 for H-FABP). Lipid peroxidation products were reported to be an independent predictor for DGF in a subgroup of the same population (Nagelschmidt et al. ${ }^{8}$ ), while IL-18 levels were highlighted by Hoogland et al. ${ }^{11}$ together with redox-active iron. In regard to PNF, FABP and LPOPs did not show any significant correlation in the MP-Trial population. On the contrary in the Maastricht population, IL-18 proved to be independent predictors of PNF (Hoogland et al.). None of these biomarkers showed significant correlation with long-term outcome in these studies, with the exception of the work by Parikh et al. ${ }^{10}$, in which L-FABP levels were modestly associated with 6-month eGFR. In one small retrospective study ${ }^{15}$, malondialdehyde (MDA), a particular lipid peroxidation product, was found to be significantly associated with DGF and 1 to 4-year post-transplant serum creatinine.

Neutrophil gelatinase-associated lipocalin. Baseline perfusate NGAL (but not post-HMP NGAL) was reported to be significantly associated with DGF in only $1 / 3$ articles without maintaining significance after multivariable analysis. Parikh et al. ${ }^{10}$ also described post-HMP NGAL levels to be modestly associated with 6-month eGFR, but not with PNF. The 
measurement time point after HMP initiation might have a crucial role in determining the efficacy of the studied biomarkers, especially when their synthesis/release could potentially be altered by hypothermia.

Single-studied biomarkers. Promising results appeared in a recent work by van Smaalen et al. ${ }^{13}$ Extracellular histone $\mathrm{H} 3$ concentration was reported to be an independent risk factor for DGF and 1-year graft survival in 390 transplant patients. As already mentioned above, nacetylglucosamine (NAG) proved to be an independent predictor for DGF in the work by Moers et al. ${ }^{7}$ Polyak et al. ${ }^{16}$, in 2000, demonstrated that iCa concentrations were significantly higher in DGF patients. Interestingly KIM-1, despite promising results as AKI biomarker, was found not to be predictive of either DGF, PNF or long term graft function in the prospective study by Parikh et al. ${ }^{10}$ A positive association with DGF was also described for matrix metalloproteinase 9 and 2 (MMP-9, MMP-2) however this analysis was performed on only 24 patients, only five of which experienced DGF. ${ }^{17}$ Guy et al. ${ }^{18}$ demonstrated a difference in perfusate metabolomic profiles of kidneys with immediate graft function and DGF. They showed that perfusate glucose, inosine, leucine and gluconate were significantly different in the DGF group with AUC 0.7-0.9, however the small size $(n=26)$ and study methodology limit the strength of these results.

\section{DCD and DBD donors}

In 10/29 articles, a mixed population of grafts from DCD and DBD donors was analysed (Table 1). When comparing DCD (or “mainly DCD”) populations versus DBD (or "mainly DBD”) populations some interesting trends emerged (Table 3). In particular, an association between high GST levels and DGF was reported in 1/4 of DCD studies but 5/5 of DBD; on the contrary the association between elevated LDH levels and DGF was reported in 5/5 of DCD studies and 2/4 of DBDs. After multivariable analysis the association GST-DGF was maintained 
only in two DBD studies, whereas the association LDH-DGF was maintained only in one DCD study. Moreover, the association to PNF largely increased when considering only DCD grafts with GST significantly associated with PNF in 6/6 articles, LDH in 2/5 articles (with maintained association after multivariable analysis) and H-FABP in 1/1 articles. On the other hand, in DBDs populations PNF was never associated with the studied biomarkers (GST 0/2, LDH 0/2, FABP $0 / 2)$.

\section{Discussion}

The present study has identified GST and LDH as the most studied and promising biomarkers to predict short-term graft function. The GSTs are a well-known enzyme family with hepatocellular activity for glutathione conjugation and the ability to bind proteins and toxins. GST elevation in the urine has been associated with renal injury and AKI in diverse clinical settings. Its isoforms alpha-GST and pi-GST are released during ischemia respectively from the

proximal and distal tubule. ${ }^{19,20}$ GST accumulation in the perfusate during kidney machine preservation could imply epithelial cell disruption and tubular damage. However, with ROC values of between $0.61-0.7$ the predictive strength was still only moderate.

LDH is a nonspecific marker of cell injury and extra-renal conditions such as circuitdependant haemolysis can determine its elevation. Numerous studies have investigated the distribution of $\mathrm{LDH}$ in normal renal tissue and its elevation in the serum and urine in patients with renal diseases. ${ }^{21,22}$ A recent experimental model combining ischemia-reperfusion injury with magnetic resonance cortical imaging, demonstrated increased LDH activity and release in the interstitial space from cells undergoing necrosis and apoptosis. ${ }^{23}$

Neutrophil gelatinase-associated lipocalin is released by renal tubular cells in response to ischemic and toxic injury and has therefore gained much attention as biomarker for AKI. Both 
urine and plasma NGAL have demonstrated to be powerful independent predictors of AKI in different clinical settings but its role in the transplant and preservation settings is still debated. ${ }^{24-}$ ${ }^{26}$ Despite this background, its use in the pre-transplant assessment has never been extensively studied and preliminary results are not encouraging.

Overall, the included studies span a long time-period, and no articles were found to be included between 1981 and 1995. For studies published prior to 1981, perfusion machines were prototypes and often required external intervention (e.g., for $\mathrm{pH}$ adjustment), standard HMP perfusion solutions were not yet available with albumin-based plasma protein fraction solutions used as perfusate. Most importantly, the definitions of outcomes were not standardized in these studies and different interpretations of the terms delayed graft function and acute tubular necrosis can be found. Lastly, it is worth considering that post-transplant care, immunosuppression and graft survival in those days was very different.

This current study appears to be the most extensive systematic review on this topic. We have summarised all the available data relating to biomarkers in kidney hypothermic machine perfusion and their association with graft outcome from 1973 to today. We selected DGF and PNF as outcomes because they were frequently compared with biomarkers in the studies and are clinically relevant. Additional factors that may also be relevant in outcomes include donor age and, in the context of DCD, warm ischaemic time. The literature is clear that increasing durations of CIT and donor age are linked to DGF and long-term graft survival. ${ }^{27}$ The range of median CIT in this study was 14-42h (median 24) which is higher than current values in the UK and Europe. In addition, delayed graft function is known to increase the risk of acute rejection and potentially decrease long-term allograft survival. Its occurrence and duration can have a negative impact on the graft outcome. ${ }^{28}$ However, recent work suggests that DGF is a different entity in DCD and DBD kidneys. The incidence of DGF in DCD kidneys does not have an impact on graft survival 
in the same way it does in DBD kidneys. The warm ischaemic insult in DCD kidneys more frequently leads to DGF and although there are higher rates of PNF, those that recover DGF don't have significantly different outcomes. However, the brainstem death cascade that leads to DGF in DBD kidneys does not appear to have the same recoverability in the long term. ${ }^{29}$

Our work suggests that elevated perfusate levels of GST, LDH and FABP are often associated with DGF. Also, IL-18 and lipid peroxidation products levels appear to be promising biomarkers in predicting delayed graft function. Despite this, recurring evidence suggests that predictive value of a single elevated biomarker is usually low or, at best, moderate. An attempted sub-analysis comparing the DBD vs DCD populations suggested some differences worth considering. In particular, GST and LDH elevation appeared more often associated with DGF among DBDs and DCDs respectively. GST elevation in DCDs was frequently associated with PNF. Despite the small number of studies, the frequent overlap between DBD and DCD patients in the same cohorts, and the lack of direct comparisons, it would be helpful to analyse these populations separately in future studies. This may be a reflection on the different pathophysiology of DGF between DCD and DBD kidneys but unfortunately the study numbers are too small to be conclusive.

The ability to predict primary non-function is perhaps more important as the outcome is devastating for the patient. Unfortunately, less than half of the included articles studied the association between biomarkers and PNF and the majority of them were not powerful enough to establish a significant association after adjusting for confounding factors. Reasons for this were the low rate of PNF, especially when grafts from DBD donors with low warm ischaemia time were considered, and the small sample sizes. GST and LDH were the most common biomarkers associated with PNF, LDH being an independent risk factor in two studies from the Maastricht population. Other independent risk factors, IL-18 and redox-active iron levels, appeared in one 
single study. As expected, in the light of the numerous variables of a transplant natural history, none of the included studies was able to demonstrate an independent statistically significant association between one single biomarker and long-term outcome with the exception of the recent work by van Smaalen et al. ${ }^{13}$

Our review has some limitations. The inclusion of different articles by the same research groups, with possible overlap between populations, was necessary so important results were not overlooked. In addition, five studies ${ }^{30-34}$ have to be considered preliminary versions of already included articles. Their results are consequently highlighted and related to the more recent publications by the same research groups in Table 1-2. Limited information was available to assess the optimum time points for assessment of biomarkers during the preservation process and this is reflected in the variation of timings of GST sampling which were between 1 and $15 \mathrm{~h}$ of HMP, which makes interpretation difficult. The included articles are graded on behalf of a quality appraisal tool for case series studies modified by Moga et al. ${ }^{6}$, and although the more recent studies were graded 'good' the majority of the others had a high risk of bias due to retrospective or non-comparative study design. Due to the heterogeneity of the included studied a metaanalysis was not performed.

Although this review focusses on HMP it is important to mention progression in the perfusion field that has not been evaluated. HMP with the addition of oxygen has recently been assessed in two clinical trials as part of the consortium for organ preservation in Europe (COPE) and (unpublished) results suggest that this may be beneficial. It would be interesting to assess biomarkers such as GST, LDH and lactate in this context as the addition of oxygen should theoretically reduce ischaemic/anaerobic injury. Ex vivo Normothermic Machine perfusion (EVNP or NMP) is a technique that involves pumping the kidney with a body temperature, blood-based, oxygenated solution. Early work by Hosgood and Nicholson suggested that DCD 
kidneys undergoing $1 \mathrm{~h}$ EVNP immediately prior to transplant had reduced rates of delayed graft function (DGF) compared with matched controls. ${ }^{35}$ This question is currently being assessed in a multicentre RCT. ${ }^{36}$ EVNP has also been used as a means of viability assessment, which has resulted in the successful transplantation of DCD kidneys declined for transplant. ${ }^{37}$ There is currently no clinical data on biomarkers of viability although this is a potentially fertile area of research. Preclinical work in discarded human kidneys undergoing 1h NMP showed urinary NGAL may be a useful measure of kidney quality. ${ }^{38}$

Prolonged periods of kidney perfusion may also be useful for viability assessment, repair and regeneration. A phase II clinical trial in deceased donor kidneys using the OrganOx kidney NMP device is due to commence in early 2020 and will assess preservation periods of up to 24h of NMP.

\section{Conclusion}

This systematic review demonstrates that no single biomarker measured during hypothermic machine perfusion is able to accurately predict short-term or long-term graft outcome. GST levels represent the most important predictor of delayed graft function, but combinations of multiple biomarkers and new biomarkers should be tested in new studies. To date, our clinical parameters

provide a footprint, but to properly assess higher risk donor organs we need a more refined finger print, which requires further study. 


\section{Figure legend}

Figure 1 - Search flowchart. 552 studies were identified by searching MEDLINE, Embase and the Transplant Library through the Ovid platform, updated to the $31^{\text {st }}$ May 2019. After duplicate removal and screening by title and abstract 60 studies remained eligible for inclusion. After fulltext revision 31 studies were excluded and 29 were included in the analysis. 


\section{References}

1. Moers C, Smits JM, Maathuis M-HJ, et al. Machine Perfusion or Cold Storage in Deceased-Donor Kidney Transplantation. N Engl J Med. 2009;360(1):7-19. doi:10.1056/NEJMoa0802289

2. Treckmann J, Moers C, Smits JM, et al. Machine perfusion versus cold storage for preservation of kidneys from expanded criteria donors after brain death. Transpl Int. 2011;24(6):548-54. doi:10.1111/j.1432-2277.2011.01232.x

3. O’Callaghan JM, Morgan RD, Knight SR, Morris PJ. Systematic review and meta-analysis of hypothermic machine perfusion versus static cold storage of kidney allografts on transplant outcomes. Br J Surg. 2013;100(8):991-1001. doi:10.1002/bjs.9169

4. $\quad$ Singh SK, Kim SJ. Epidemiology of kidney discard from expanded criteria donors undergoing donation after circulatory death. Clin J Am Soc Nephrol. 2016;11(2):317-323. doi:10.2215/CJN.07190715

5. Bhangoo RS, Hall IE, Reese PP, Parikh CR. Deceased-donor kidney perfusate and urine biomarkers for kidney allograft outcomes: a systematic review. Nephrol Dial Transplant Off Publ Eur Dial Transpl Assoc - Eur Ren Assoc. 2012;27(8):3305-3314. doi:10.1093/ndt/gfr806

6. Institute of Health Economics. https://www.ihe.ca/advanced-search/development-of-aquality-appraisal-tool-for-case-series-studies-using-a-modified-delphi-technique. Accessed November 28, 2018.

7. Moers C, Varnav OC, Van Heurn E, et al. The value of machine perfusion perfusate biomarkers for predicting kidney transplant outcome. Transplantation. 2010;90(9):966-73. doi:10.1097/TP.0b013e3181f5c40c

8. Nagelschmidt M, Minor T, Gallinat A, et al. Lipid peroxidation products in machine perfusion of older donor kidneys. J Surg Res. 2013;180(2):337-42. doi:10.1016/j.jss.2012.04.071

9. Hall IE, Bhangoo RS, Reese PP, et al. Glutathione S-transferase iso-enzymes in perfusate from pumped kidneys are associated with delayed graft function. Am J Transplant. 2014;14(4):886-96. doi:10.1111/ajt.12635

10. Parikh CR, Hall IE, Bhangoo RS, et al. Associations of Perfusate Biomarkers and Pump Parameters with Delayed Graft Function and Deceased Donor Kidney Allograft Function. 
Am J Transplant. 2016;16(5):1526-39. doi:10.1111/ajt.13655

11. Pieter Hoogland ER, De Vries EE, Christiaans MHL, Winkens B, Snoeijs MGJ, Ernest Van Heurn LW. The value of machine perfusion biomarker concentration in DCD kidney transplantations. Transplantation. 2013;95(4):603-10. doi:10.1097/TP.0b013e31827908e6

12. De Vries B, Snoeijs MGJ, Von Bonsdorff L, Ernest Van Heurn LW, Parkkinen J, Buurman WA. Redox-active iron released during machine perfusion predicts viability of ischemically injured deceased donor kidneys. Am J Transplant. 2006;6(11):2686-93. doi:10.1111/j.1600-6143.2006.01510.x

13. Van Smaalen TC, Beurskens DMH, Hoogland ERP, et al. Presence of Cytotoxic Extracellular Histones in Machine Perfusate of Donation after Circulatory Death Kidneys. Transplantation. 2017;101(4):e93-e101. doi:10.1097/TP.0000000000001590

14. Cho SI, Zalneraitis B, Ohmi N, Arias IM. Prediction of cadaver kidney function by ligandin analysis. J Surg Res. 1981;30(4):361-4. doi:10.1016/0022-4804(81)90172-4

15. Kosieradzki M, Kuczynska J, Piwowarska J, et al. Prognostic significance of free radicals: mediated injury occurring in the kidney donor. Transplantation. 2003;75(8):1221-1227.

16. Polyak MMR, Arrington BOM, Stubenbord WT, et al. The influence of pulsatile preservation on renal transplantation in the 1990s. Transplantation. 2000;69(2):249-58. doi:10.1097/00007890-200001270-00010

17. Moser MAJ, Arcand S, Lin H Bin, et al. Protection of the transplant kidney from preservation injury by inhibition of matrix metalloproteinases. PLoS One. 2016;11(6):e0157508. doi:10.1371/journal.pone.0157508

18. Guy AJ, Nath J, Cobbold M, et al. Metabolomic analysis of perfusate during hypothermic machine perfusion of human cadaveric kidneys. Transplantation. 2015;99(4):754-9. doi:10.1097/TP.0000000000000398

19. McMahon BA, Koyner JL, Murray PT. Urinary glutathione S-transferases in the pathogenesis and diagnostic evaluation of acute kidney injury following cardiac surgery: A critical review. Curr Opin Crit Care. 2010;16(6):550-5. doi:10.1097/MCC.0b013e32833fdd9a

20. Vanmassenhove J, Vanholder R, Nagler E, Van Biesen W. Urinary and serum biomarkers for the diagnosis of acute kidney injury: an in-depth review of the literature. Nephrol Dial Transplant Off Publ Eur Dial Transpl Assoc - Eur Ren Assoc. 2013;28(2):254-273. doi:10.1093/ndt/gfs380 
21. Kang SK, Ha CY, Cho KH, Park SK, Kim UH. Changes of lactate dehydrogenase and its isoenzyme activity in renal diseases. Nephron. 1991;57(1):55-59. doi:10.1159/000186216

22. Zager RA, Johnson ACM, Becker K. Renal cortical lactate dehydrogenase: A useful, accurate, quantitative marker of in vivo tubular injury and acute renal failure. PLoS One. 2013;8(6):e66776. doi:10.1371/journal.pone.0066776

23. Nielsen PM, Laustsen C, Bertelsen LB, et al. In situ lactate dehydrogenase activity: a novel renal cortical imaging biomarker of tubular injury? Am J Physiol Renal Physiol. 2017;312(3):F465-F473. doi:10.1152/ajprenal.00561.2015

24. Mishra J, Dent C, Tarabishi R, et al. Neutrophil gelatinase-associated lipocalin (NGAL) as a biomarker for acute renal injury after cardiac surgery. Lancet. 2005;365(9466):1231-8. doi:10.1016/S0140-6736(05)74811-X

25. Devarajan P. Neutrophil gelatinase-associated lipocalin (NGAL): A new marker of kidney disease. Scand J Clin Lab Invest. 2008;68(sup241):89-94. doi:10.1080/00365510802150158

26. Mårtensson J, Bellomo RR. The rise and fall of NGAL in acute kidney injury. Blood Purif. 2014;37(4):304-10. doi:10.1159/000364937

27. Summers DM, Johnson RJ, Allen J, et al. Analysis of factors that affect outcome after transplantation of kidneys donated after cardiac death in the UK: a cohort study. Lancet. 2010;376(9749):1303-11. doi:10.1016/S0140-6736(10)60827-6

28. Ojo AO, Wolfe RA, Held PJ, Port FK, Schmouder RL. Delayed graft function: Risk factors and implications for renal allograft survival. Transplantation. 1997;63(7):968-74. doi:10.1097/00007890-199704150-00011

29. de Kok MJ, McGuinness D, Shiels PG, et al. The neglectable impact of delayed graft function on long-term graft survival in kidneys donated after circulatory death associates with superior organ resilience. Ann Surg. 2019;270(5):877-883. doi:10.1097/SLA.0000000000003515

30. Kwiatkowski A, Danielewicz R, Kosieradzki M, et al. Six-year experience in continuous hypothermic pulsatile perfusion kidney preservation. In: Transplant Proc. 2001;33(12):913-5. doi:10.1016/S0041-1345(00)02267-3

31. Polyak MMR, Arrington BOM, Kapur S, Stubenbord WT, Kinkhabwala M. Calcium ion concentration of machine perfusate predicts early graft function in expanded criteria donor kidneys. Transpl Int. 1999;12(5):378-82. doi:10.1007/s001470050243 
32. Kievit JK, Oomen APA, Janssen MA, van Kreel BK, Heineman E, Kootstra G. Viability assessment of non-heart-beating donor kidneys by alpha glutathione S-transferase in the machine perfusate. Transplant Proc. 1997;29(1-2):1381-3. doi:10.1016/s00411345(96)00603-3

33. Kievit JK, Nederstigt AP, Oomen APA, Janssen MA, Schoot L, Kootstra G. Release of $\alpha$ glutathione S-transferase ( $\alpha \mathrm{GST}$ ) and $\pi$-glutathione S-transferase ( $\pi \mathrm{GST}$ ) from ischemic damaged kidneys into the machine perfusate - relevance to viability assessment. Transplant Proc. 1997;29(8):3591-3. doi:10.1016/S0041-1345(97)01036-1

34. Daemen JH, Heineman E, Kootstra G. Viability assessment of non-heart-beating donor kidneys during machine preservation. Transplant Proc. 1995;27(5):2906-2908.

35. Nicholson ML, Hosgood SA. Renal transplantation after ex vivo normothermic perfusion: the first clinical study. Am J Transplant. 2013;13(5):1246-52. doi:10.1111/ajt.12179

36. Hosgood SA, Saeb-Parsy K, Wilson C, et al. Protocol of a randomised controlled, openlabel trial of ex vivo normothermic perfusion versus static cold storage in donation after circulatory death renal transplantation. BMJ Open. 2017;7(1):e012237. doi:10.1136/bmjopen-2016-012237

37. Hosgood SA, Thompson E, Moore T, et al. Normothermic machine perfusion for the assessment and transplantation of declined human kidneys from donation after circulatory death donors. Br J Surg. 2018;105(4):388-394. doi:10.1002/bjs.10733

38. Hosgood SA, Nicholson ML. An assessment of urinary biomarkers in a series of declined human kidneys measured during Ex Vivo Normothermic Kidney Perfusion. Transplantation. 2017;101(9):2120-2125. doi:10.1097/TP.0000000000001504

39. Sevinc M, Stamp S, Ling J, Carter N, Talbot D, Sheerin N. Ex vivo perfusion characteristics of donation after cardiac death kidneys predict long-term graft survival. Transplant Proc. 2016;48(10):3251-3260. doi:10.1016/j.transproceed.2016.09.049

40. Khalid U, Ablorsu E, Szabo L, et al. MicroRNA-21 (miR-21) expression in hypothermic machine perfusate may be predictive of early outcomes in kidney transplantation. Clin Transplant. 2016;30(2):99-104. doi:10.1111/ctr.12679

41. Gok MA, Pelzers M, Glatz JFC, et al. Do tissue damage biomarkers used to assess machine-perfused NHBD kidneys predict long-term renal function post-transplant? Clin Chim Acta. 2003;338(1-2):33-43. doi:10.1016/j.cccn.2003.07.023

42. Kosieradzki M, Danielewicz R, Kwiatkowski A, et al. Early function of kidneys stored by 
continuous hypothermic pulsatile perfusion can be predicted using a new "viability index." Transplant Proc. 2002;34(2):541-3. doi:10.1016/s0041-1345(01)02838-x

43. Polak W, Danielewicz R, Kwiatkowski A, et al. Pretransplant evaluation of renal viability by glutathione S-transferase in machine perfusate. Transplant Proc. 2000;32(1):171-2. doi:10.1016/S0041-1345(99)00921-5

44. Kootstra G, Daemen JH. The non-heart-beating donor. Transplant Proc. 1996;28(1):16.

45. Daemen JWHC, Oomen APA, Janssen MA, et al. Glutathione s-transferase as predictor of functional outcome in transplantation of machine-preserved non-heart-beating donor kidneys. Transplantation. 1997;63(1):89-93. doi:10.1097/00007890-199701150-00017

46. Horpácsy G, Scholz D, Althaus P, May G. Clinical experiences in the gambro-preservation unit: analysis of 101 human cadaver kidneys. Eur Surg Res. 1979;11(1):50-60. doi:10.1159/000128051

47. Burleson RL, Jones DB, Yenikomshian AM, Cornwall C, Devoe C, Derito J. Clinical renal preservation by cryoperfusion with an albumin perfusate: renal perfusion with albumin. Arch Surg. 1978;113(6):688-92. doi:10.1001/archsurg.1978.01370180030003

48. Modgill VK, Wiggins PA, Rosenberg IL, Humphrey CS, Giles GR. An evaluation of viability tests of human cadaveric kidneys. Br J Surg. 1977;64(8):548-53. doi:10.1002/bjs.1800640806

49. Baxby K, Johnson RWG. Prediction of kidney viability before transplantation. Br J Surg. 1975;62(10):810-2. doi:10.1002/bjs.1800621015

50. Johnson RWG, Anderson M, Taylor RMR, Swinney J. Significance of perfusate lactic acidosis in cadaveric renal transplantation. Br Med J. 1973;1(5850):391-5. doi:10.1136/bmj.1.5850.391 